\title{
THE INDICATOR AS AN AID TO ECONOMY.
}

By Professon W. E. DALBY, F.R.S., Member of Council.

Chairman: Dr. T. E. Stanton, C.B.E., Member of Council.

[Abridged.]

In the absence of the Author, the Paper was presented by Professor F. F. D. Witcheld, Member.

The indicator diagram gives valuable information about the timing of the cycle of operations and about valve-setting, and this is as important as the determination of horse-power. In quick running internal-combustion engines a mere fraction of a second difference in timing makes a large difference in the power developed, and the quickest and best way of obtaining the proper setting of an engine is by means of an indicator. For indicating engines in which pressure changes are rapid, the moving parts of the instrument must be reduced to the smallest possible mass in order to avoid inertia errors, and even then the movements must be small. With such limitations, diagrams of convenient size can most easily be obtained optically. Optical indicators may be divided into two types: (1) the piston type, and (2) the disk or diaphragm type. The piston type was developed by the late Professor Hopkinson, and subsequently by Professor Burstall. The disk type is represented in the Carpentier instrument and in the indicator designed by the Author, also in the indicator designed by the late Professor Watson.

The Carpentier indicator has one mirror receiving two motions at right angles to each other, one proportional to pressure, and other to the stroke, whereas in the Dalby-Watson indicator two mirrors are provided, one for each motion. When used with quick-running engines, the spot of light, reflected on to a ground-glass screen, travels so quickly that the eye sees a complete indicator diagram traced out in light, and the slightest variation in the conditions in the cylinder is instantaneously seen in the change of shape of the diagram.

[THE I.MeCh.E.] 
The objection to the diaphragm indicator is that the diaphragm gets hot and slightly changes its scale, but this disadvantage is outweighed by the many advantages which such an indicator possesses.

The use of the indicator as an aid to economy was illustrated by a set of five half-plate diagrams which showed the additional work and improved economy obtained from a gas-engine running at 250 r.p.m. by simply advancing the spark. Another set of three diagrams showed the improved economy obtained by spark advancement in a petrol motor running at 1,200 r.p.m. Diagrams from a Diesel engine were also shown, and a method of investigating the phenomenon of " after-burning" was outlined.

\section{Discussion.}

Mr. Druite Halpin referred to the determination of power by using indicators, and to the laborious process of planimetering the cards. This labour could be avoided by using an integrating indicator which served the double purpose of an indicator and a planimeter.

Professor F. W. Bunstall said that in 1893 he had used a Carpentier indicator, and whilst finding it useful as a qualitative instrument, he decided it was worthless for quantitative work. $\mathrm{He}$ had found that the diaphragms changed their scales and suffered from hysteresis. In 1917 he began to construct an indicator for experimental quantitative work, and after investigating Professor Hopkinson's indicator, he considered that the advantages outweighed the drawbacks. The drawbacks were considerable, inasmuch as there was piston friction, considerable inertia, and the question of cooling the cylinder; but they could be faced and overcome.

His first indicator was constructed to work up to 400 r.p.m., but during the last eighteen months inertia of moving parts had been reduced, and the spring improved, until the final form, which was on exhibition in the Institution, had been reached. It was small and portable, and being all hardened and water-cooled, he had not hesitated to screw it straight on to a petrol motor and let it work all the time the engine was running. The piston could be lubricated without taking it out. The inertia of the disk indicator was less than that of the piston type, but the calculated periodic time for his piston type was small, being only $\frac{1}{1450}$ second, and the indicator could work up to 2,500 r.p.m. 
Another advantage of the optical indicator was the facility it offered for the taking of light spring cards. Such diagrams had shown that the conditions in the induction pipes of many motorcars were terrible. With regard to Diesel and high-compression Crossley engines, optical indicators were the only kind that could be relied upon. He had known pencil indicators register pressures. $100 \mathrm{lb}$. per square inch in excess of the pressures actually existing.

Mr. J. L. Hodeson asked what was the amount of inertia pressure in the optical indicator, and to what extent the gases were throttled in passing to the indicator. Also to what degree did hysteresis affect the accuracy of the diagram

Dr. J. G. STewaRT said that he saw no reason for using a piston in an indicator intended for workshop practice for petrol engines. Dirt on the piston caused considerable friction, and it was probable that no piston indicator had yet been designed in which friction had been eliminated. Determination of friction at atmospheric pressure gave no indication of friction at considerable pressures. In diaphragm indicators there was a difference of temperature on the two sides of the diaphragm which could not be repeated when calibrating the instrument.

Mr. J. Ornus said that on stationary internal-combustion engines he had found the pencil indicator satisfactory up to 300 r.p.m. In practice, if the mechanical efficiency of an Otto cycle engine came out below 80 per cent or above 90 per cent, the accuracy of the indicator was doubted. If the efficiency fell between these values, the result was considered good or indifferent as the value was above or below the normal. As both types of indicator were calibrated under static pressure conditions, he suggested there was no satisfactory proof that the optical was more accurate than the pencil indicator at speeds below 300 r.p.m. For high-speed work the optical indicator was undoubtedly the only type. Against its extended use was the difficulty of attaching it to multi-cylinder engines with closed crank-cases.

Professor E. F. D. WITCHELL, in reply, said that accuracy in the pencil indicator depended largely on the person who took the card. Apart from the friction of the mechanism, there was friction of the pencil on the paper, which might be very great in unskilled hands But below 300 r.p.m. the pencil indicator appeared satisfactory. He would not be expected to hold a brief for the disk type of optical 
indicator as against the piston type, but personally he considered the friction which occurred in the piston type as very serious. He had noticed that in some diagrams which Professor Burstall was exhibiting, the compression lines, which presumably should be smooth curves, appeared as a series of flats. That change in the character of the curve was probably due to friction, the magnitude of which was unknown, and for which therefore correction could not be made. On the other hand the alteration in strength of a diaphragm due to heat had been estimated by the late Dr. Watson to be from 2 to $2 \frac{1}{2}$ per cent, and allowance could be made for it. He had not found hysteresis very great in these instruments, and as regarded calibration, the diagrams shown had been calibrated in $50 \mathrm{lb}$. steps, while the indicator was still in position. For that purpose a Crosby pressure-gauge tester had been used. 